\title{
ON BASE RADICAL THEORY IN FINITE SETTINGS
}

\author{
LAUREN THORNTON ${ }^{\circledR}$
}

(Received 4 February 2020; first published online 13 March 2020)

2010 Mathematics subject classification: primary 16N80; secondary 17A65.

Keywords and phrases: base radical class, base semisimple class, operator semigroup.

This thesis is an exploration of base radical theory for universal classes of algebras first introduced by Puczyłowski. Results are applicable for the theory in settings like groups, rings, loops and hoops. Much of the investigation is restricted to the finite case to allow duality to play a greater role.

Class operators are employed to study radical properties based on the factor and accessible structure of these algebras, rather than the traditional use of element properties. Two semigroups formed by distinct compositions of the operators are identified by a complete listing of their elements. A number of operator compositions will generate a radical class for all subclasses of the universal class. These radical classes can be placed in a lattice by containment.

Several conditions for factor and accessible properties that the algebras may possess which determine the order of the operator semigroup are given. Class operator equalities are used to prove a number of structural properties for universal class elements. Operator representations of hereditary radical classes and factor closed semisimple classes are described using weaker conditions than factor closed and hereditary for the generating class. Further, a subclass of finite algebras need only be hereditary and closed under extensions to be a semisimple class.

In the setting of finite associative rings, we show that the largest hereditary subclass of a radical class is a radical-semisimple class. This can be dualised, and the largest homomorphically closed subclass of a semisimple class is also a radical-semisimple class. On the structure of a finite associative ring, we prove that every simple homomorphic image of an accessible subring is a simple accessible subring of a homomorphic image.

Here, where Kurosh-Amitsur and base radical classes coincide, the largest hereditary class contained in a Kurosh-Amitsur radical class is a Kurosh-Amitsur

Thesis submitted to the University of the Sunshine Coast in March 2019; degree approved on 6 November 2019; supervisor Robert McDougall.

(C) 2020 Australian Mathematical Publishing Association Inc. 
radical class. We show by counterexample in the setting of algebras of the Puczyłowski type that the result does not hold when 'Kurosh-Amitsur' is replaced by 'base'.

The operator approach is then used to compare and contrast Kurosh-Amitsur and base radical constructions. An operator description of a class that is always KuroshAmitsur radical and base semisimple but not necessarily Kurosh-Amitsur semisimple or base radical is described, and a necessary and sufficient condition is given which identifies the Kurosh-Amitsur radical classes that are base radical.

Parts of this research have been published in [1-3].

\section{References}

[1] R. G. McDougall and L. K. Thornton, 'On base radical operators for classes of finite associative rings', Bull. Aust. Math. Soc. 98(2) (2018), 239-250.

[2] R. G. McDougall and L. K. Thornton, 'A comparison of Kurosh-Amitsur and base radical classes' (under review).

[3] L. K. Thornton, 'On base radical and semisimple operators for a class of finite algebras', Beitr. Algebra Geom. 59(2) (2018), 361-374.

\section{LAUREN THORNTON, Department of Mathematics,}

School of Science and Engineering, University of the Sunshine Coast,

Queensland, Australia

e-mail: lauren.thornton@ research.usc.edu.au 